\title{
SPECTROSCOPY OF STELLAR CORONAL SOURCES WITH THE MEDIUM ENERGY EXPERIMENT ON EXOSAT
}

\author{
R. Pallavicini ${ }^{1}$, L. Pasquini ${ }^{2}$, J.H.M.M. Schmitt ${ }^{2}$ and G. Tagliaferri ${ }^{3}$ \\ 1 Arcetri Astrophysical Observatory, Florence, ltaly \\ 2 Max-Planck-Institut fur Extraterrestrische Physik, Garching, Germany \\ 3 ESTEC, EXOSAT Observatory, Noordwijk, The Netherlands
}

ABSTRACT. We summarize some of the results obtained by spectral analysis of EXOSAT ME observations of stellar coronal sources. We focus on time-resolved spectroscopy of stellar flares and on the determination of the temperature structure of quiescent RS CVn binaries

\section{INTRODUCTION}

The Medium Energy (ME) experiment on the EXOSAT Observatory provided pulseheight spectra of stellar $X$-ray sources over the range $\approx 1-10 \mathrm{KeV}$ with a resolution $E / \Delta E \approx 3-5$. This resolution does not allow individual lines to be resolved, but is already sufficient to determine the general shape of the spectrum and to search for the presence of the Iron line complex at $6.7 \mathrm{KeV}$. By fitting the observed spectrum with appropriate theoretical models, it is possible to derive information on physical parameters such as electron temperature and emission measure. Broad-band data over the spectral range $0.05-2 \mathrm{KeV}$ were acquired simultaneously by the Low Energy (LE) experiment on EXOSAT in selected passbands. The LE data can be used in conjunction with the ME spectral data to constraint better the temperature stratification in the source. Details on the ME and LE experiments on EXOSAT can be found in White and Peacock (1988).

Stellar coronal data obtained with the EXOSAT ME include:

- quiescent emission from RS CVn and Algol-type binaries (about 15 sources);

- quiescent and/or flaring emission from a few other very active late-type stars (flare stars, PMS stars, etc; in total, about 5 sources);

- time-resolved spectra of flares in M dwarts and RS CVn binaries (about 10 sources).

As can be seen from the list above, the limited sensitivity of the ME experiment allowed only a small number of stellar coronal sources to be studied spectroscopically at energies higher than $\approx 1 \mathrm{KeV}$. Nevertheless, these data are important because they give us a first glimpse of what could be obtained with higher sensitivity and higher spectral resolution; moreover, they have provided new interesting results which have increased appreciably our understanding of stellar coronal structure and variability. In this paper we will give some examples of the results that can be obtained from the analysis of ME data, focussing on time-resolved spectroscopy of stellar flares and on spectral analysis of quiescent emission from RS CVn binaries. Results of eclipse observations of binaries are reported by $\mathrm{N}$. White elsewhere in this volume. 


\section{TIME-RESOLVED SPECTROSCOPY OF STELLAR FLARES.}

The highly eccentric orbit of the EXOSAT Observatory allowed continuous monitoring of a source for periods as long as three days. This is particularly useful when studying time varying phenomena such as flares or when monitoring light modulations associated with orbital and/or rotational motions. Several flares were observed by EXOSAT that were sufficiently intense and long-lived to allow time-resolved spectroscopy during their evolution.

Probably the best example is the five hours-long flare observed from Algol on August 19, 1983 , which was first studied by White et al. (1983). The flare started at $\approx 10: 00$ UT, peaked at $\approx$ 11:00 UT and than decayed steadily until $\approx 15: 00$ UT. We have reanalyzed this flare by subdividing it into five time intervals, one during the rise phase (from 10:00 to 11:00 UT), and the other four during the decay. ME spectra were accumulated over these five time intervals, after subtraction of a preflare quiescent spectrum. The emissivity model assumed was that of an optically-thin line+continuum thermal plasma as computed by Mewe, Gronenschild and van den Oord (1985). We find that the temperature was decreasing from a peak value of $(6.1 \pm 0.8) \times 10^{7} \mathrm{~K}$ to $(2.3 \pm 0.6) \times 10^{7} \mathrm{~K}$ in the late decay. During the same time interval the volume emission measure decreased from $6.7 \times 10^{53} \mathrm{~cm}^{-3}$ to $1.1 \times 10^{53} \mathrm{~cm}^{-3}$. The average temperature during the rise phase (from 10:00 to 11:00 UT) was $(5.2 \pm 0.8) \times 10^{7} \mathrm{~K}$ and the emission measure was $3.0 \times 10^{53} \mathrm{~cm}^{-3}$. Although the count rate was not sufficiently high to resolve the temperature variations during the rise phase, these data already indicate that the plasma was first heating and then cooling during the flare evolution. The total energy released over the spectral band $0.1-10$ $\mathrm{KeV}$ was $1.1 \times 10^{35}$ ergs.

Another flare for which time-resolved spectra could be obtained throughout the flare was observed from the M dwarf flare star EQ Peg on August 6,1985 . Only the peak and the decay phase were observed in this case, from $\approx 06: 30$ UT, when the flare reached its maximum intensity, to $\approx 09: 00$ UT, when the emission returned to the quiescent level. Over this time interval, five different spectra could be accumulated that were fitted with a simple bremsstrahlung spectrum + a Fe line at $6.7 \mathrm{KeV}$. This model spectrum provided a good fit to the data, with a reduced $\chi^{2}$ which was in all cases less than 1 . Except for the last spectrum, obtained during the very late decay, the presence of an Fe line complex at $6.7 \mathrm{KeV}$ appears necessary to tit the data satisfactorily. The same line complex was also prominent in the spectra of the Algol flare discussed above. During the decay of the EQ Peg flare, the temperature was found to decrease from $(4.4 \pm 0.4) \times 10^{7} \mathrm{~K}$ to $(1.7 \pm 0.4) \times 10^{7} \mathrm{~K}$ and the emission measure decreased from $2.1 \mathrm{x}$ $10^{53} \mathrm{~cm}^{-3}$ to $3.0 \times 10^{52} \mathrm{~cm}^{-3}$. The total energy released by the flare during the observed period over the spectral band $0.1-10 \mathrm{KeV}$ was $\approx 5 \times 10^{33} \mathrm{ergs}$.

There are only a few other flares observed by EXOSAT from $M$ dwarf stars that could be analyzed spectrally at different times during their evolution. The best case is another long-lived event observed from the M dwarf eclipsing binary YY Gem on Nov 14, 1983. The temperature appeared to decrease slightly from $3.3 \times 10^{7} \mathrm{~K}$ at the flare peak to $2.4 \times 10^{7} \mathrm{~K}$ in the late decay, while the emission measure decreased over the same time interval from $1.8 \times 10^{53} \mathrm{~cm}^{-3}$ to $4.5 \mathrm{x}$ $10^{52} \mathrm{~cm}^{-3}$. In all other cases only one or two spectra could be accumulated over the duration of the flare. The derived temperatures are in the range $\approx 2 \times 10^{7} \mathrm{~K}$ and $\approx 4 \times 10^{7} \mathrm{~K}$, similar to typical temperatures observed in X-ray flares on the Sun. We stress, however, that the data sample is quite small: in addition to the two flares mentioned above (from EQ Peg and YY Gem), there were only three other flares from $M$ dwart stars for which the $M E$ experiment could detect enough photons to allow reliable spectral fits: they were observed from the stars Wolf 630 (on Aug 25, 1985), AT Mic (on May 25, 1985) and again from EQ Peg (on Aug 29, 1984). Details of spectral fits of these flares will be given elsewhere (Pallavicini, Stella and Tagliaferri 1988). 


\section{QUIESCENT EMISSION FROM RS CVN STARS}

A class of stellar sources which was more easy to observe with the EXOSAT ME is constituted by the RS CVn binaries. For these sources, it was possible to observe not only flares, but also quiescent emission. The count rates for the closest and brightest sources were high enough to allow meaningful spectral fits to be performed (this is usually not the case for quiescent emission of $M$ dwarfs, which were either not detected by the ME during quiescent periods or were too faint to allow spectral analysis).

For each RS CVn star observed by EXOSAT we have analyzed at least one ME spectrum (Pasquini, Schmitt and Pallavicini 1988). The LE data obtained simultaneously in one or more soft $X$-ray filters were used in conjunction with the ME spectral data in order to determine the temperature stratification in the source. The data were fitted using a Raymond and Smith (1977) line + continuum thermal model and a fixed interstellar hydrogen column density (estimated for each source from its distance and the average value of the hydrogen density in the solar neighborhood given by Paresce 1984). We used one- and two-temperature thermal models and we evaluated the goodness of the fit by the usual $\chi^{2}$ technique. The results (which refer to a sample of fifteen sources) can be summarized as follows. For spectra of sufficiently high $\mathrm{S} / \mathrm{N}$ ratio, a two-temperature model is required to simultaneously fit the ME and LE data. The derived temperatures cluster around two well defined values, the lower one at $\approx 6-8 \times 10^{6} \mathrm{~K}$, and the upper one at about $2-3 \times 10^{7} \mathrm{~K}$. Only spectra with a low $\mathrm{S} / \mathrm{N}$ ratio could be fitted satisfactorily with a one-temperature model.

The above results are similar to those obtained previously with the SSS and IPC experiments on board the EINSTEIN Observatory (Swank et al. 1981, Majer et al. 1986). However, as discussed by Majer et al. (1986) and Schmitt et al. (1988), an acceptable fit with a two-temperature model does not necessarily imply the existence in the source of two physically distinct regions in two different temperature regimes. MonteCarlo simulations of EINSTEIN IPC and EXOSAT ME spectral data (Pasquini, Schmitt and Pallavicini 1988) show in fact that acceptable two-temperature fits could also be obtained with a continuous emission measure distribution, i.e. with the presence of plasma at all temperatures from less than $10^{6} \mathrm{~K}$ to several times $10^{7} \mathrm{~K}$. Such a continuous emission measure distribution could result from the presence of magnetically confined structures in the coronae of these stars.

In order to investigate whether the coronae of RS CVn binaries could be interpreted as constituted by a single family of coronal loops of the solar-type (i.e. in hydrostatic equilibrium and energy balance) we have fitted all spectra with a continuous emission measure distribution of the simple form $E M \sim\left(T / T_{M}\right)^{\alpha}$, where $T_{M}$ is the maximum temperature in the loop and $\alpha$ is the slope of the distribution which in the solar case is expected to be $\approx 1$. We found that this simple model is capable of fitting some of our RS CVn data, but not all of them. For instance, while we were able to fit with a continuous emission measure distribution the quiescent LE + ME spectrum of UX Ari, AR Lac, II Peg and Algol, no acceptable fit could be obtained for Capella, $\lambda$ And, $\sigma \mathrm{CrB}$ and HR 1099. Moreover, even for those sources that were fitted with a continuous emission measure distribution, the derived $\alpha$ values were usually larger than expected from simple models of solar coronal loops.

The above findings indicate that substantial differences are likely to exist between the coronae of RS CVn stars and the coronae of the Sun and other single late-type stars. The results of our spectral fitting analysis suggest that either the coronae of most RS CVn stars involve more than one family of loops (as also suggested by eclipse observations, cf. White et al. 1988) or the coronal structures in these stars have a more complex emission measure distribution than the simple power-law assumed by us. 


\section{OTHER CORONAL SOURCES}

The EXOSAT data base comprises a few other coronal sources for which spectral data could be obtained with the ME experiment. One of the most interesting observations is a flare from the A-type visual binary Castor (Pallavicini et al. 1988). This source has a nearby $M$ dwart flare star companion (YY Gem) and the two souces cannot be spatially resolved by the ME experiment. However, by comparing the ME light curve with the separate light curves of YY Gem and Castor obtained simultaneously by the LE experiment, it is possible to attribute unambiguously the observed flare events to either YY Gem or Castor. A flare from the latter source was detected on Nov 14, 1984 at 03:40 UT. Spectral analysis of the flare was done over three distinct time intervals throughout the flare evolution (peak, early decay, late decay). The results of the spectral fits, made using the optically-thin line+continuum thermal model of Mewe, Gronenschild and van den Oord (1985), shows that the temperature decreased from $\approx 50 \times 10^{6} \mathrm{~K}$ at the flare peak to $\approx 30 \times 10^{6} \mathrm{~K}$ in the late decay, while the emission measure decreased from $5.0 \times 10^{53} \mathrm{~cm}^{-3}$ to 1.2 $\times 10^{53} \mathrm{~cm}^{-3}$. The total energy released by the flare over the spectral band $0.1-10 \mathrm{KeV}$ was $4.3 \times$ $10^{33}$ ergs. The time scales and energy involved as well as the values of the derived parameters are similar to those typically observed from $\mathrm{M}$ dwarf flare stars. As discussed by Pallavicini et al. (1988) this suggests the possibility that the flare originated not from the A-type primary components of the Castor system, but rather from an unseen low-mass companion.

Another interesting case is a serendipitous stellar source that was observed to flare in the LE and ME detectors while EXOSAT was pointing to the Seyfert galaxy III ZW 2 (Tagliaferri et al. 1988). Since the source was quite faint, only one ME spectrum could be accumulated during the flare, yielding an average temperature of $\approx 4.5 \times 10^{6} \mathrm{~K}$ and an emission measure of $1.8 \times 10^{54}$ $\mathrm{cm}^{-3}$. The total energy released by the flare in X-rays was $1.9 \times 10^{35}$ ergs, much larger than for typical flares observed in $M$ dwarf stars and comparable to the total energy released by the flare on Algol. Tagliaferri et al. (1988) have argued that the flare likely originated from the secondary component of the visual binary HD 560 (a system formed by a B9V primary and a GOVe secondary). From optical studies HD $560 \mathrm{~B}$ has been identified as a post-T Tauri star. This optical identification is consistent with the high quiescent $X$-ray luminosity $\left(\approx 10^{30} \mathrm{erg} \mathrm{s}^{-1}\right)$ and strong flaring activity observed from this source.

\section{REFERENCES}

Majer, P., Schmitt, J.H.M.M., Golub, L., Harnden, F.R.Jr. and Rosner, R.: 1986, Ap. J. $300,360$. Mewe, R., Gronenschild, E.H.B.M. and van den Oord, G.H.: 1985, Astr. Ap. Suppl. 62, 197.

Pallavicini, R., Stella, L. and Tagliaferri, G.: 1988, to be submitted to Astr. Ap.

Pallavicini, R., Tagliaferri, G., Pollock, A., Schmitt, J.H.M.M. and Rosso, C.: 1988, submitted to Astr. Ap.

Paresce, F.: 1984, Astr. J. 89,1022.

Pasquini, L., Schmitt, J.H.M.M. and Pallavicini, R.: 1988, submitted to Astr. Ap.

Raymond, J.C. and Smith, B.W.: 1977, Ap. J. Suppl. 35, 419.

Schmitt, J.H.M.M., Pallavicini, R., Monsignori-Fossi, B.C. and Harnden, F.R.Jr.: 1987, Astr. Ap. $179,197$.

Swank, J.H., White, N.E., Holt, S.S. and Becker, R.H.: 1981, Ap. J. 246, 208.

Tagliaferri, G., Giommi, P., Angelini, L., Osborne, J.P. and Pallavicini, R.: 1988, Ap. J. Letters 331, L113.

White, N.E. et al. : 1988, this volume.

White, N.E., Culhane, J.L., Parmar, A.N., Kellet, B.J., Kahn, S., van den Oord, G.H.J. and Kuipers, J.: 1986, Ap. J.301, 262.

White, N.E. and Peacock, A.: 1988, in X-ray Astronomy with EXOSAT (R. Pallavicini and N.E. White eds. ), in press. 